\title{
General-Uebersicht der Helophorinen Europas und der angrenzenden Gebiete.
}

Von A. Kuwert in Wernsdorf (Ostpreussen).

\section{Vorbemerkungen.}

Die Eintheilung der Helophorus-Arten in 5 Gruppen ist nach einem auffälligen, natürlichen Modus erfolgt. Da das Genus Empleurus Hope einerseits nicht ganz mit dem nachfolgend aufgestellten Subgenus Trichohelophorus zusammenfällt, andererseits das sich den Anforderungen des Hope'schen Genus genau unterstellende Subgenus Kyphohelophorus ganz ausser Acht lässt, so musste, um nicht ganz nahestehende Thiere von einander zu reissen, der ursprïnglich für eine sehr geringe Artenzahl gegründete Namen Empleurus für das die Subgenera Kyphohelophorus und Trichohelophorus umfassende Genus adoptirt und die Hope'schen Gattungskennzeichen entsprechend erweitert werden.

Die reiche Gruppe Rhopalohelophorus ist sehr schwierig, weil die Punktirung der Flügeldecken und die Form der Intervalle überall variirt und der Bau der Tarsen bei den meisten Arten conform bleibt. Ebenso ist die Färbung sehr variabel und bietet oft kein sicheres Unterscheidungsmerkmal. Hierzu kommt, dass das wahrscheinlich sicherste Kennzeichen der einzelnen Arten, die faserartigen Haare aus den Tarsengelenken und Schienen wegen ihrer unglaublichen Fragilität kaum an irgend einem Stücke vollkommen vorhanden sich zeigen - (am allerwenigsten bei geklebten Stücken, bei welchen sie fast immer verloren gehen) - und deshalb für die Bestimmung werthlos werden. Es musste deshalb besonders auf die äusserliche Form des Körpers, vorzüglich des Thorax zurückgegriffen werden.

Man wird mir vielleicht zum Vorwurfe machen, dass ich durch beschleunigte Veröffentlichung vermeintlich neuer Arten einige Synonyme geschaffen habe. Schuld hieran war einerseits die vollständige Unzugänglichkeit der Beschreibungen mancher Arten durch die Herren Autoren, andererseits die gewaltige Zerstreutheit der einschlägigen Literatur. Auch hatte ich von der R ey'schen Concurrenzarbeit, soweit sie die frauzösischen Thiere betrifft, keine Ahnung und bin - fast nach Beendigung meiner Arbeit - durch dieselbe überrascht worden.

In jedem Falle jedoch haben meine Veröffentlichungen den gewünschten Zweck erreicht, mir ein sehr grosses Material vor Augen zu führen.

"Wiener Entomologische Zeitung" V. Heft 6. (20. Juli 1886.) 
Sehr unterstützt wurde meine Untersuchung, was ich hier dankend hervorbeben will, durch das theilweise oder gesammte Sammlungsmaterial, sowie das freundliche Entgegenkommen der Herren Avillarius in Stockholm, Bergroth in Helsingfors, Bellier de la Chavignerie in Evreux, Dohrn in Stettin, Eppelsheim in Grünstadt, von Heyden in Bockenheim, Krüper in Athen, Miller in Wien, Nickerl in Prag, Ragusa in Palermo, Régimbart in Evreux, Reitter in Mödling, Sahlberg in Helsingfors, S chneider in Dresden, von Seidlitz in Rathshof, Stussiner in Laibach u. a. m. Den typischen, Motschulskyschen Hel. elongatus konnte ich nicht erhalten, doch dürfte er sicher mit dem von mir dafür gehaltenen Thiere zusammenfallen, neben granularis L. zu stehen kommen und richtig placirt sein. Von den übrigen 66 Species haben mir nur 4 nicht vorgelegen: baicalicus Motsch., parallelus Motsch., villosus Duftsch. und crenatus Rey. Die Existenz von villosus Duftsch. muss ich bezweifeln. Ebenso gut, als Stïcke von aquaticus L. mit einem abgekürzten Punktstreifen von 2 Punkten vorkommen, wo die gewöhnliche Zahl 4 bis 8 beträgt, und wie mir ein Stück von strigifrons (!!) mit einem einseitigen abgekürzten Punktstreifen von 2 Punkten (von Berlin als aequalis) zugeschickt wurde, kann es auch einen einzelnen aquaticus L. var. villosus Küster ohne abgekürzten Punktstreifen oder eine ähnliche Verbindung eines andern Thiers gegeben haben.

Der letzte Berliner Katalog enthält nur 32 Arten, von welchen mehrere als solche eingehen mussten, punientanus Schauf. als Varietät zu filitarsis Schauf., mirabilis Miller, weil nach der eigenen Aussage des genannten Autors dies Thier nie existirt hat, nie beschrieben ist, tigrinus Schaum als Synonym zu subcostatus Kolen., creticus Kiesw., weil ich ihn wegen der zahllosen Uebergänge nur für eine Varietät von griseus Hbst. halten konnte u. s. w. Dagegen waren unter dem vermeintlichen Synonym obscurus Muls. Rey und aeneipennis Thoms. mehrere Thiere vereinigt, die ich trennen zu müssen glaubte, da sie zum Theil wohl nicht richtig erkannt waren.

\section{General-Uebersicht.}

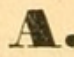

Die abwechselnden Intervalle der Flügeldecken sind entweder höckerartig aufgetrieben oder stark kielförmig erhoben, im letzten Fall mit oben rückwärts gekrümmten Hakenbörstchen 
besetzt. Der Aussenrand der Flügeldecken tritt als eine fast immer oder doch meistens bis zur Naht reichende Leiste vor. Die Flügeldecken haben stets einen abgekürzten Punktstreifen neben der Naht. Die Augen sind meistentheils mit dem Clypeum zusammen abgerundet. Die mittelsten Tarsenglieder, zumal der Vorder- und Mittelfüsse, sind, von oben gesehen, fast immer kurz perlförmig. Aus der Oberseite der Tarsengelenke wachsen entweder keine oder kurze stärkere Borsten und nicht die Fasern oder Wimpern der Abtheilung B. Die Käfer leben weniger im Wasser, sondern mehr in dem Auswurf am Ufer und in der Nähe der Gewässer, meistens mit starker Schmutzkruste bedeckt.

Genus: Empleurus. (Hope in partibus.)

A. Mit gehöckerten Flügeldecken .

\section{Subgen. Kyphohelophorus.}

Halsschildvorderecken stark und flach ausgebreitet, Käfer länger. - Dauria, Sibiria.

1. baicalicus Motsch. 1860. Halsschildvorderecken nicht stark ausgebreitet, Käfer kürzer. Fennia. Suecia. Baltia.

2. tuberculatus Gyll. 1808.

B. Mit abwechselnd stark kielförmig erhabenen Zwischenräumen der Punktstreifen auf den Flügeldecken und einer Reihe aufrechtstehender und am oberen Ende rückwärts gekrümmter Hakenbörstchen auf den Kielen; mit meistens spindelförmigem Endglied der Palpen. II. Subgen. Trichohelophorus. ๙) Die Mittelwülste des Thorax hinter dem Vorderrande tief eingedrückt.

a) Schulterecken der Elytren mehr oder weniger winklig oder spitz vorgezogen.

1. Flügeldecken gelb oder gelbbraun, glänzend mit schwarzen Querzeichnungen. (Empleurus Hope.) Dalmatien, Pyrenäen, Algier, Balearen, Sicilien, Balcan. - (fennicus Payk. 1798). $\quad 3$ rugosus Oliv 1789.

2. Der ganze Käfer schwarz oder schwarzbraun, wenig glänzend, mit sehr breit vorgezogenem Flügelrande und gröberer Beborstung. (Empleurus Hope) - Sicilien.

4. siculus Kuw. 1886.

b) Schulterecken abgerundet rechteckig; Käfer braungelb, mit geringen dunkeln Zeichnungen. Grösser (Empleurus Hope) - Pyrenäen, Algier. 5. pjrenaeus Kuw. *) 1885.

*) Ist vielleicht nur Varietät von rugosus, was späteren Ermittelungen vorbehalten bleiben muss. 
ß) Die Mittelwülste des Thorax sind hinter dem Vorderrande nicht tief eingedrückt.

a) Halsschildhinterecken stumpfwinklig.

1. Augen mit dem Clypeum zusammen abgerundet. Flügelrandfalte bis zur Naht reichend. Mittlere Tarsenglieder kurz. Halsschild kurz; Grösse von rugosus Ol. Form von nubilus Fabr. (Empleurus Hope) - Algier, Pyrenäen.

6. aper Pandellé 1877.

Halsschild länger; Form von rugosus (Empleurus Hope). Balearen, Pyrenäen, Gall. mer., Dalmatien.

7. porculus Bevé 1881 .

2. Augen vorspringend, nicht mit dem Vorderkopf zusammen abgerundet. Flügelrandfalte nicht bis zur Naht reichend. Mittlere Tarsenglieder etwas länger. Halsschildwulste gekörnt. Käfer meistens dunkler braun. Gall. mer., Hispan., Graecia. 8. alternans*) Gené 1839. Halsschildmittelwulste glatt. Käfer heller braun. Balcan, Gall. mer., Graecia, Corfu.

var. intermedius Muls. 1844.

b) Aeusserste Halsschildhinterecken nicht deutlich stumpfwinklig, ziemlich rechteckig.

1. Mit höhlenartiger Unterbrechung der 2. Rippe in der Nähe des Schildchen. Augen mit dem Kopfschild abgerundet. (Empleurus Hopes) Alpes, Italia, Hispania, Helvetia (Simplon). - (alpinus Hen. 1841. fracticostis Fairm. 1859.)

9. Schmidtii Villa 1838.

2. Ohne höhlenartige Unterbrechung der 2. Rippe in der Nähe des Schildchens.

๙) Augen mit dem Clypeum abgerundet.

1. Käfer $2 \cdot 3 \mathrm{~mm}$ mit etwas gewölbterem Halsschilde (Empleurus Hope). Euphrat, Asia minor., Dalmatia.

10. Mesopotamiae Kuw. 1886.

2. Käfer 3 bis $3.2 \mathrm{~mm}$ mit flacherem Halsschilde.

a) Kürzer, mit hinter der Mitte verbreiterten Flügeldecken (Empleurus Hope). Eur. bor. et med.

11. nubilus Fbr. 1775.

b) Länger, mit hinter der Mitte nicht verbreiteten Flügeldecken. Eur. mer., Crimea. - (meridionalis Motsch. 1860).

var. costatus Goeze.

*) Diese Species bildet den Uebergang zwischen Empleurus und Helophorus. 
ß) Augen, von vorne gesehen, deutlich vorspringend. Halsschild mit sehr breiter Mittelrinne. Die 1. und 2. Rippe von der Naht vereinigen sich (immer?) vor ihrem Ende. Käfer schmal. Dalmat. - (linearis Kuw.) 1885.

12. lineellus Kuw.*) 1886.

c) Mit deutlich weniger als rechtwinkligen oder sichtlich spitzwinkligen Halsschildhinterecken. Der aufgebogene Flügelrand reicht meistens nicht bis zur Flügelnaht. 1. Käfer schmal; Grösse wenig über nubilus, Augen mit dem Kopfschild abgerundet. Kaukasus.

13. tessellatus Klug in litt.

2. Käfer breiter und grösser. Augen schwach vorspringend.

๙) Hinterleib nicht stark zugespitzt verschmälert.

a) Mit purpurglänzendem Halsschilde. Käfer wenig befilzt, blank. Kaukasus, Balkan, Graecia. - (opalisans Bessa, acutipalpis Muls. 1852).

14. micans Fald. 1835.

b) Ohne purpurglänzenden Halsschild; stark grau befilzt; der Filz auf den Rippen häufig getigert, fleckenartig schwarz unterbrochen. Balkan, Asia min., Graecia. - (tigrinus Schaum in litt.)

var. subcostatus Kolen. 1846.

ß) Hinterleib am Ende stark zuspitzend abgerundet. Der Halsschild vor den Hinterecken sehr tief ausgeschnitten. Algier.

15. oxygonus Bedel 1881.

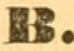

Die abwechselnden Intervalle der Flügeldecken sind weder höckerartig aufgetrieben, noch gleichzeitig kielförmig und mit Hakenborsten besetzt. Die Furchen der Flügeldecken laufen in den Hinterrand derselben. Die Augen sind nicht mit dem Clypeum zusammen abgerundet. Aus der ()berseite der Gelenke der Tarsenglieder entspringen fast immer 1 oder 2 lange (häufig fehlende) Faserhaare oder Wimpern, welche sich über die Mitte der Tarsen fortlegen. Die Käfer leben fast ausschliesslich im Wasser und werden nur selten ausserhalb desselben angetroffen.

Genus. Helophorus Fabr.

\section{I.}

Mit abgekürztem Punktstreifen neben dem Schildchen.

*) Heloph. linearis ist bereits in Amerika vorhanden, weshalb ich den Namen in lineellus verwandelte. 
C. Mit gleichen oder abwechselnd schwach erhabenen Zwischenräumen der Flügeldecken, mit oder ohne wahrnehmbare Behaarung sämmtlicher Zwischenräume. Endglied der Palpen meistens nicht spindelförmig. Grössere Thiere.

III. Subgen. Megahelophorus.

a) Das 2. und 4. Intervall von der Naht deutlich erhaben, von dem 6. nur das vordere Ende.

1. Mit röthlicher Hinterleibsspitze und breiterer Figur.

๙) Halsschildseitenrand vor den Hinterecken nicht ausgeschnitten. Eur. bor., Lappon. - (Gyllenhalli Sahlb.) 1875.

16. fennicus Gyll. 1808.

ß) Halsschild stark ausgeschnitten. Eur. arct.

var. borealis Thoms. 1860 .

2. Mit rothem Hinterleibe; jeder Ring mit schwarzer Querlinie. Schmälere Figur. Sibir.

var. sibiricus Motsch. 1860.

3. Mit ganz schwarzem Hinterleibe und höhern Flügelrippen; schmälere, kleinere Figur. Dovre, Norwegen.

var. dovrensis Kuw. 1886.

Hierher gehört auch (der Motchulsky'schen Beschreibung nach) der mir unbekannt gebliebene, hinten nicht verschmälerte, tief punktirt gestreifte, mit grossen Punkten zwischen den gewölbten Intervallen versehene, aus der Kirgisensteppe stammende :

17. parallelus Motsch. 1860.

b) Die abwechselnden Intervalle sind kaum oder nicht gewölbter als die andern.

1. Die Halsschildwülste sind deutlich nablig gekörnt.

๙) Hinterleib kürzer; Käfer dunkel metallisch. Masc. $5 \mathrm{~mm}$, fem. $9 \mathrm{~mm}$. Flügeldecken schwach reihig behaart. Europa, As. bor. - (grandis Illig.) 18. aquaticus Lin.

ß) Hinterleib länger. Halsschild und Kopf sehr stark goldglänzend. Halsschildseitenrand hinten schwach ausgeschnitten. Flügeldecken in Reihen stärker behaart. Masc. et fem. $9 \mathrm{~mm}$, gleich gross. Corfu, Graecia, Italia, Sicilia. - (villosus Küster) 1850.

var. Milleri Kuw. 1886.

$\gamma)$ Wie der vorige, doch weniger behaart; die hinteren Halsschildseitenränder stärker ausgeschnitten. Masc. 5, fem. $9 \mathrm{~mm}$ lang. Syria, Palästina.

var. syriacus Kuw. 1885. 
2. Die Halsschildwülste sind auf der Mitte desselben nicht deutlich gekörnt, doch nicht spiegelblank.

ж) Vorderkopf und Stirne oben ganz metallisch. (Nach Thomson der letzte Hinterleibsring nicht gekerbt.) $4 \mathrm{~mm}$. Abwechselnde Intervalle nicht erhaben. Eur. bor. et alp.

var. aequalis Thoms. 1867.

ק) Kopf und Halsschild dunkelbraun, metallisch, Flügeldecken rothbraun. Sib. occ. 19. Bergrothi Sahlb. 1875.

3. Halsschildwülste spiegelblank, höchstens sehr fein punktirt.

a) Alle Reihen der Flügeldecken aus sehr feinen Punkten. Käfer schmäler, flacher. Hispan., Pyren.

20. frigidus Graells. 1846.

ß) Käfer breiter, gewölbter. Alle Reihen aus grossen Punkten. Sib. occ.

21. niger Sahlb. 1875.

\section{II.}

Ohne abgekürzten Punktstreifen neben dem Schildehen.

D. Mit spindelförmigem Endgliede der Palpen (d. h. die Längsachse des Palpengliedes ist gleichzeitig überall der Mittelpunkt der Querschnitte). IV. Subgen. Atractohelophorus.

1. Flügeldecken kurz, stopplig behaart (nach Duftschmidt*)

Käfer gross, $9 \mathrm{~mm}$. Linz. 22. villosus Dufts. 1805-25.

2. Flügeldecken nicht deutlich behaart, Käfer kleiner.

๙) Abwechselnde $Z$ wischenräume der Flügeldecken deutlich erhabener. Diese gekerbt, gestreift, braungelb mit Zeichnungen.

1. Halsschildwülste gewölbt.

a) Halsschild nach vorne verbreitert. Käfer sehr undeutlich mit kleinen Börstchen behaart. Britannia, Gallia, Germania. 23. arvernicus Muls. 1844.

b) Halsschild nach vorne verschmälert. Sibiria orient. - (consimilis Manerh. 1853).

24. inquinatus Manerh. 1852.

2. Halsschildwülste nicht gewölbt. Die Halsschildfurchen nur Linien. Dalmat.

25. singularis Mill. 1879.

ß) Abwechselnde Zwischenräume der Flügeldecken erhabener; diese punktirt gestreift.

*) Das ron D uftschmidt beschriebene Thier ist mir nicht zugänglich gewesen, dürfte jedoch sicher zu stellen sein. 
Halsschildwülste stark gewölbt. Käfer fast einfarbig dunkel. Alp. Eur., Kaukasus. - (tristis Ullrich in litt.)

26. nivalis Giraud, Miller 1852.

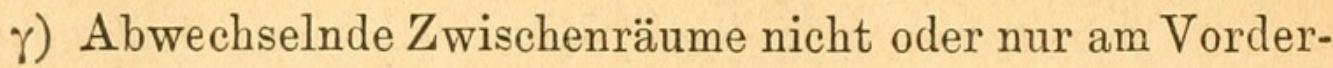
rande deutlich erhabener.

1. Käfer stets dunkel metallisch.

a) Mit metallglänzenden Palpen und Beinen. Alp. centr., Norwegen. - (nivalis Thoms.) 27.glacialis Villa 1833.

b) Mit nur metallglänzendem Palpenendgliede.

๙) Mit grosser Mittelgrube des Halsschildes und am Vorderrande deutlich abwechselnd erhabenen Intervallen der Flügeldecken. Kaukasus, Balkan. - ( maculatus Motsch. 1860. - caucasicus Kuw. 1885).

28. guttulus Motsch. 1860.

ß) Ohne Mittelgrube, mit ganz ebenen, glatten Intervallen der Flïgeldecken. Corsica.

29. insularis Reiche 1861.

2. Käfer mit helleren Flügeldecken; höchstens in den Variationen dunkler; mit nicht oder selten metallischem Palpenendgliede.

a) Flügeldecken gekerbt-gestreift; gelb mit Zeichnungen. Sib. occ., Kauk. 30. orientalis Motsch. 1860.

b) Flügeldecken punktirt-gestreift.

๙) Palpenendglied dick spindlig; Käfer kürzer. Flügeldecken braungrau mit Zeichnungen. Eur. bor. et med. - (granularis Thoms. 1867).

31. griseus Hbst. 1785, Er. 1839.

§) Palpenendglied spitz spindlig. Käfer länger. Flügeldecken braungrau mit Zeichnungen. Punktstreifen oft ohne vertiefte Linie. Eur. mer. et med., Kaukasus, Persia.

var. brevipalpis Bedel 1867.

$\gamma)$ Palpenendglied scharf spitzig. Käfer ganz dunkel metallgrün, mit 2 hellen Punkten vor der Flügelspitze. Croatien, Montenegro. var. montenegrinus Kuw. 1885.

ঠ) Palpenendglied spitzig. Flügeldecken gelb mit dunkeln Zeichnungen. Halsschild gleichmässig fein gekörnt. Punktreihen meist in nicht vertieften Linien. Graecia, Smyrna, Hispania, Sicilia. var. creticus Kies. 1858. 


\section{$2 \mathrm{BHL}$ Biodiversity Heritage Library}

Kuwert, August Ferdinand. 1886. "General-Uebersicht der Helophorinen

Europas und der angrenzenden Gebiete." Wiener entomologische Zeitung 5, 221-228. https://doi.org/10.5962/bhl.part.20588.

View This Item Online: https://www.biodiversitylibrary.org/item/43771

DOI: https://doi.org/10.5962/bhl.part.20588

Permalink: $\underline{\text { https://www.biodiversitylibrary.org/partpdf/20588 }}$

\section{Holding Institution}

Smithsonian Libraries

\section{Sponsored by}

Smithsonian

\section{Copyright \& Reuse}

Copyright Status: NOT_IN_COPYRIGHT

This document was created from content at the Biodiversity Heritage Library, the world's largest open access digital library for biodiversity literature and archives. Visit BHL at https://www.biodiversitylibrary.org. 